\title{
A method for verifying liveness of protocols modeled as a class of ECFSM
}

\author{
M. Higuchi, S. Sugawa, and M. Fujii \\ Dept. of Information and Computer Sciences, Osaka University \\ Toyonaka, Osaka 560, Japan
}

\begin{abstract}
In a previous work, we proposed a method for verifying safety of communication protocols modeled as two extended communicating finite-state machines with two unbounded FIFO channels connecting them. This paper presents a method for verifying liveness based on the above method. Liveness is formulated as $Q$-liveness which states " $\forall g s \in R S \exists g s^{\prime} \in G S_{Q}\left\{g s^{\prime}\right.$ is reachable from $\left.g s\right\}$ ", where $R S$ and $G S_{Q}$ denote the set of reachable global states and the set of global states satisfying the property $Q$, respectively. In the proposed verification method, a finite degenerated reachability graph, $D R G$, of a given protocol is constructed. In $D R G$, each node represents a subset of reachable states, and if there exists an edge from a node $v_{i}$ to another node $v_{j}$, where $v_{i}$ and $v_{j}$ represent subsets of reachable states $R S_{i}$ and $R S_{j}$ respectively, then " $\forall g s \in R S_{i} \exists g s^{\prime} \in R S_{j}\left\{g s^{\prime}\right.$ is reachable from $\left.g s\right\}$ " holds. By exploring $D R G, Q$-liveness is shown to hold. An experimental result on verifying liveness of a sample protocol extracted from the data transfer phase of the OSI session protocol, is also described to show the effectiveness of the verification method.
\end{abstract}

Keyword Codes: C.2.2;D.2.4

Keywords: Network Protocol; Program Verification

\section{Introduction}

For communication protocols, safety and liveness are considered as fundamental properties, and methods for verifying those properties have been studied to obtain reliable communication systems ${ }^{[1]}$. In general, the safety property states that the protocol never falls into any undesirable state, and the liveness property states that some desirable event will eventually occur. As an instance of desirable events, we can consider "receptions of some specified message" or "transitions to normal termination state".

2-CFSMs (resp. 2-ECFSMs) is a protocol model consisting of two communicating finite-state machines (resp. two extended communicating finite-state machines) and bilateral FIFO queues connecting them. A state of an extended communicating finite-state machine is expressed by not only a value of finite control but also context variables whose 
ranges of values are potentially infinite. If the boundedness of the communication channels of 2-CFSMs is guaranteed, the set of global states is finite and therefore many important properties, including safety and liveness, are decidable in principle ${ }^{[2]}$. Some decision procedures have been proposed ${ }^{[3][4]}$ for channel-bounded 2-CFSMs based on state exploration which enumerates reachable global states. However, there exist many practical protocols whose state spaces are infinite because of the unboundedness of communication channels or infinite state space of individual protocol machines. Since both non-channel-bounded 2CFSMs and a two counter machine, which is a subclass of single ECFSM, are known to be universal ${ }^{[2][5]}$, traditional state exploration techniques cannot be used for such a class. For non-channel-bounded CFSMs, several verification methods were proposed ${ }^{[6][7][8]}$. Among them, only [8] discussed a method for verifying liveness.

On the other hand, as a method for verifying safety of non-channel-bounded 2ECFSMs, we proposed a verification method via invariant ${ }^{[9]}[10]$ under the assumption that context variables are restricted to integral registers and the operations on integral registers are restricted to substitution, addition and comparison of two values. In spite of the assumption, a wide variety of practical protocols which use "sequence numbers", can be modeled as 2-ECFSMs. On the other hand, since a class of two-counter machines is still a subclass of such restricted ECFSM, the expressive power of such restricted 2-ECFSMs is much higher than 2-CFSMs. The verification method proposed in ${ }^{[9][10]}$ is summarized as follows:

(a) A human verifier describes a logical formula, say $F$, on global states which is expected to satisfy (i) $R S \subseteq G S(F)$ and (ii) $G S(F) \subseteq S A F E$, where $R S, G S(F)$ and $S A F E$ denote the reachable global states, the set of global states satisfying $F$ and the set of safe global states, respectively.

(b) A verification system automatically shows the above (i) and (ii) by structural induction on event sequences.

In this paper, a method for verifying liveness of 2-ECFSMs is proposed by extending the above method for verifying safety. Liveness property is formulated as $Q$-liveness which states " $\forall g s \in R S \exists g s^{\prime} \in G S_{Q}\left\{g s^{\prime}\right.$ is reachable from $\left.g s\right\}$ ", where $R S$ and $G S_{Q}$ denote the set of reachable states and the set of global states satisfying the property $Q$, respectively. In the proposing verification method of liveness, a finite degenerated reachability graph $D R G$ of a given protocol is constructed. In $D R G$, each node $v_{i}$ represents a subset $R S_{i}$ of reachable states. For each $R S_{i}$, all states in $R S_{i}$ share common properties concerned with the followings:

- the states of the finite controls of the protocol machines,

- characteristics of message type sequences in the channels (e.g. " $A \cdot A^{*}$ ", one or more type $A$ messages),

- characteristics of parameters of messages in the channels (e.g. they are arranged in strictly increasing order), and

- relations between the values of the context variables of the protocol machines and parameters of messages in the channels.

The verification method for safety in [9] gives a way to define such subsets. In $D R G$, if there exists an edge from a node $v_{i}$ to another node $v_{j}$, then " $\forall g s \in R S_{i} \exists g s^{\prime} \in$ $R S_{j}\left\{g s^{\prime}\right.$ is reachable from $\left.g s\right\}$ " holds. By exploring $D R G, Q$-liveness is shown to hold. 
The rest of this paper is organized as follows: Section 2 defines a class of 2-ECFSMs. Section 3 summarizes a verification method of safety, proposed in [9]. In section 4, we define $Q$-liveness and degenerated reachability graphs, and give a method for constructing a degenerated reachability graph $D R G$. Also in section 4 , we discuss another restriction on protocol model to obtain an efficient verification procedure. In section 5 , a verification system and an experimental result on verifying liveness of a sample protocol are described.

\section{Protocol Model}

Two extended communicating finite-state machines (2-ECFSMs) are a protocol model which consists of two extended communicating finite-state machines and two unbounded FIFO channels connecting them. Formally, it is defined as follows.

Definition 1 A protocol machine $P M$ is a 4-tuple $(S, \Sigma, T, S I)$, where

(M1) $S=\langle S F, r\rangle$ defines a set of states, where $S F$ is the state set of the finite control part of the machine and $r$ is the number of registers (context variables) which store nonnegative integers. Let $\mathcal{N}$ denote the set of nonnegative integers. The state space of the protocol machine is $S F \times \mathcal{N}^{r}$.

(M2) $\Sigma=\Sigma_{-} \cup \Sigma_{+}$: a finite set of message types. $\Sigma_{-}$is a set of message types which $P M$ can send and $\Sigma_{+}$is a set of message types which $P M$ can receive. $\Sigma_{-}$and $\Sigma_{+}$ are supposed to be disjoint. For $d \in \Sigma$ and $n \in \mathcal{N},\langle d, n\rangle$ is called a message and $n$ is called the parameter of the message. The number of parameters of a message is assumed to be exactly one only for simplicity. In the following, for a message sequence $u, \operatorname{type}(u)$ and parameter $(u)$ denote the message type sequence and the parameter sequence of $u$, respectively. The set of events $E V$ of the protocol machine is defined in connection with the set of messages sent or received by the machine, i.e. $E V=\left\{\langle d, n\rangle \mid d \in \Sigma_{-}, n \in \mathcal{N}\right\} \quad \cup \quad\left\{\langle d, n\rangle \mid d \in \Sigma_{+}, n \in \mathcal{N}\right\}$. The former subset is the set of sending events and the latter one is the set of receiving events. To explicitly express that the event $\langle d, n\rangle$ is a sending (or receiving) event, we may write $-\langle d, n\rangle($ or $+\langle d, n\rangle)$.

(M3) $T$ : a set of actions. An action $\left(s f, d, s f^{\prime}, C, R\right)$ is a 5-tuple defining a set of transitions, where $s f \in S F, d \in \Sigma, s f^{\prime} \in S F$. C, called an action condition, is a simultaneous inequality to be satisfied by the parameter value $n$ of the outgoing or incoming message and the register values $p_{1}, p_{2}, \ldots, p_{r}$ of the protocol machine at the state just before the action. $R$, called a register modification function is a partial function from $\mathcal{N}^{r} \times \mathcal{N}$ to $\mathcal{N}^{r}$ defining the changes of register values in the transition. For a state $s=\left(s f c, p_{1}, p_{2}, \ldots, p_{r}\right)$ of the protocol machine and an action $\left(s f, d, s f^{\prime}, C, R\right) \in T$, if $s f c=s f$ and $p_{1}, p_{2}, \ldots, p_{r}, n$ satisfy $C$, then the protocol machine can send or receive (according to $d$ in $\Sigma_{-}$or $\Sigma_{+}$) the message $\langle d, n\rangle$ at the state $s$ and then the machine transits to $s^{\prime}=\left(s f^{\prime}, R\left(p_{1}, p_{2}, \ldots, p_{r}, n\right)\right)$. A nondeterministic state transition function $\delta$ from $S F \times \mathcal{N}^{r} \times E V$ to $S F \times \mathcal{N}^{r}$ is determined by $T$ as follows, where $s=\left(s f, p_{1}, p_{2}, \ldots, p_{r}\right)$ and $e=\langle d, n\rangle$ :

$$
\begin{aligned}
\delta(s, e)= & \left\{\left(s f^{\prime}, R\left(p_{1}, p_{2}, \ldots, p_{r}, n\right)\right) \mid\right. \\
& \left.\left(s f, d, s f^{\prime}, C, R\right) \in T \text { and } p_{1}, p_{2}, \ldots p_{r}, n \text { satisfies } C\right\},
\end{aligned}
$$


For $s \in S F \times \mathcal{N}^{r}$ and $e \in E V$, if $\delta(s, e)$ is not empty, then the event $e$ is said to be executable in the state $s$.

(M4) $S I \subseteq S F \times \mathcal{N}^{r}$ : a set of initial states.

Definition 2 For two protocol machines $P M_{A}=\left(\left\langle S F_{A}, r_{A}\right\rangle, \Sigma_{A}, T_{A}, S I_{A}\right)$ and $P M_{B}=$ $\left(\left\langle S F_{B}, r_{B}\right\rangle, \Sigma_{B}, T_{B}, S I_{B}\right)$, if $\Sigma_{B-}=\Sigma_{A+}$ (denoted by $\left.\Sigma_{B A}\right)$ and $\Sigma_{A-}=\Sigma_{B+}$ (denoted by $\left.\Sigma_{A B}\right), \Pi=\left(P M_{A}, P M_{B}\right)$ is called a protocol. A 4-tuple $\left\langle s_{A}, s_{B}, u_{B A}, u_{A B}\right\rangle \in\left\langle S F_{A} \times\right.$ $\left.\mathcal{N}^{r_{A}}, S F_{B} \times \mathcal{N}^{r_{B}},\left\langle\Sigma_{B A} \times \mathcal{N}\right\rangle^{*},\left\langle\Sigma_{A B} \times \mathcal{N}\right\rangle^{*}\right\rangle$ is called a global state of the protocol $\Pi . u_{B A}$ and $u_{A B}$ denote message sequences in the channels from $P M_{B}$ to $P M_{A}$ and from $P M_{A}$ to $P M_{B}$ respectively. A global state $\left\langle s i_{A}, s i_{B}, \varepsilon, \varepsilon\right\rangle(\varepsilon$ is the empty sequence) is called an initial global state of $\Pi$, when $s i_{A} \in S I_{A}$, and $s i_{B} \in S I_{B}$, or when $\left\langle s i_{A}, s i_{B}\right\rangle$ is in a specified subset of $S I_{A} \times S I_{B}$ if such a subset is explicitly specified.

Definition 3 A global state $g s^{\prime}=\left\langle s_{A}^{\prime}, s_{B}^{\prime}, u_{B A}^{\prime}, u_{A B}^{\prime}\right\rangle$ is said to be directly reachable from $g s=\left\langle s_{A}, s_{B}, u_{B A}, u_{A B}\right\rangle$ (denoted by $g s \rightarrow g s^{\prime}$ ) if one of the following conditions is satisfied for some $d \in \Sigma_{B A} \cup \Sigma_{A B}$ and $n \in \mathcal{N}$.

$$
\begin{aligned}
& s_{A}^{\prime} \in \delta_{A}\left(s_{A},-\langle d, n\rangle\right), s_{B}^{\prime}=s_{B}, u_{B A}^{\prime}=u_{B A}, \text { and } u_{A B}^{\prime}=u_{A B} \cdot\langle d, n\rangle ; \\
& s_{A}^{\prime} \in \delta_{A}\left(s_{A},+\langle d, n\rangle\right), s_{B}^{\prime}=s_{B},\langle d, n\rangle \cdot u_{B A}^{\prime}=u_{B A}, \text { and } u_{A B}^{\prime}=u_{A B} ; \\
& s_{A}^{\prime}=s_{A}, s_{B}^{\prime} \in \delta_{B}\left(s_{B},-\langle d, n\rangle\right), u_{B A}^{\prime}=u_{B A} \cdot\langle d, n\rangle, \text { and } u_{A B}^{\prime}=u_{A B} \\
& s_{A}^{\prime}=s_{A}, s_{B}^{\prime} \in \delta_{B}\left(s_{B},+\langle d, n\rangle\right), u_{B A}^{\prime}=u_{B A}, \text { and }\langle d, n\rangle \cdot u_{A B}^{\prime}=u_{A B} .
\end{aligned}
$$

If one of the former (or the latter) two conditions holds, then $P M_{A}$ (or $P M_{B}$ ) is called the performer, the relation is also denoted $g s \rightarrow_{A}^{\langle d, n\rangle} g s^{\prime}$ (or $g s \rightarrow_{B}^{\langle d, n\rangle} g s^{\prime}$ ), and $(\langle d, n\rangle, A)$ (or $(\langle d, n\rangle, B))$ is said to be executable in $g s$. The reflexive transitive closure of the relation " $\rightarrow$ " is denoted by $\stackrel{*}{\rightarrow}$ ". If $g s \stackrel{*}{\rightarrow} g s^{\prime}$, then $g s^{\prime}$ is said to be reachable from $g s$.

For a protocol $\Pi=\left(P M_{A}, P M_{B}\right)$, the set of all reachable global states from some initial global state is called the reachability set of $\Pi$.

\section{A Method for Verifying Safety}

The verification method for liveness proposed in this paper is based on the method for verifying safety proposed in [9]. Before describing the method for verifying liveness, we will review the method for verifying safety.

For a protocol $\Pi$, if a logical formula $F$ holds for every reachable global state, $F$ is said to be an invariant in $\Pi$. Let $G S(P)$ denote the set of global states satisfying a formula $P$. In the verification method, the verification system shows that (a) a logical formula $F$, described by a human verifier, is an invariant in a protocol $\Pi$, and (b) $G S(F)$ does not contain any unsafe state. If (a) and (b) hold, we can conclude that $\Pi$ is safe.

\subsection{Safety Property}

If the reachability set of a protocol $\Pi$ does not contain the following states, $\Pi$ is said to be safe:

- A global state $g s=\left\langle s_{A}, s_{B}, u_{B A}, u_{A B}\right\rangle$ is called a deadlock state if $u_{B A}=u_{A B}=\varepsilon$ and any sending event is executable in neither $s_{A}$ nor $s_{B}$; 
- A global state $g s=\left\langle s_{A}, s_{B}, u_{B A}, u_{A B}\right\rangle$ is called an unspecified reception state if either $u_{B A} \neq \varepsilon$ and $\delta_{A}\left(s_{A}\right.$, thead $\left.\left(u_{B A}\right)\right)$ is undefined, or $u_{A B} \neq \varepsilon$ and $\delta_{B}\left(s_{B},+\right.$ head $\left.\left(u_{A B}\right)\right)$ is undefined, where for a nonempty sequence $\alpha$, head $(\alpha)$ denotes the first element of $\alpha$.

A deadlock state and an unspecified reception state are called unsafe states.

\subsection{Description of a Logical Formula}

In what follows, assume that a given formula $F$ is in a disjunctive normal form $F=$ $P_{1} \vee P_{2} \vee \cdots \vee P_{n}$. Each disjunct $P_{i}$ of the formula $F$ is a conjunction of atomic formulae (or simply atoms) of the following four types. An example of $P_{i}$ is found in Table 4.

(AF1) A pair of finite states $\left\langle s f_{A}, s f_{B}\right\rangle$, where $s f_{A} \in S F_{A}$ and $s f_{B} \in S F_{B}$, is an atom which holds for a global state $\left\langle s_{A}, s_{B}, u_{B A}, u_{A B}\right\rangle$ iff the finite control parts of $s_{A}$ and $s_{B}$ are $s f_{A}$ and $s f_{B}$ respectively. We assume that exactly one AF1 type atom appears in each disjunct $P_{i}$.

(AF2) A pair of predicates $\left\langle P_{B A}, P_{A B}\right\rangle$ to specify sets of message type sequences in two communication channels is an atom which holds for a global state

$\left\langle s_{A}, s_{B}, u_{B A}, u_{A B}\right\rangle$ iff $P_{B A}$ holds for $u_{B A}$ and $P_{A B}$ holds for $u_{A B}$. Such a predicate may be defined by a regular expression. For instance, an AF2 type atom $\langle\varepsilon$, MIP . $\left.\mathrm{MIP}^{*}\right\rangle$ states that type $\left(u_{B A}\right) \in L(\varepsilon)$ and type $\left(u_{A B}\right) \in L\left(\mathrm{MIP} \cdot \mathrm{MIP}^{*}\right)$, where $L(r)$ denotes the set of sequences expressed by the regular expression $r$. We also assume that exactly one AF2 type atom appears in each $P_{i}$.

(AF3) For a message sequence $u$ and a set of message types $\Sigma_{X}, \operatorname{subseq}\left(u, \Sigma_{X}\right)$ denotes the subsequence of $u$ whose message types are restricted to $\Sigma_{X}$. A predicate on a message parameter sequence of $\operatorname{subseq}\left(u_{B A}, \Sigma_{X}\right)$ (or subseq $\left(u_{A B}, \Sigma_{X}\right)$ ) for some $\Sigma_{X}$ is also an atom. For instance, "step $1\left(\operatorname{subseq}\left(u_{A B},\{\mathrm{MIP}\}\right)\right)$ " states that the parameter sequence parameter $\left(\operatorname{subseq}\left(u_{A B},\{\right.\right.$ MIP $\left.\left.\}\right)\right)$ satisfies the predicate "step1". By "step 1 " we mean that the parameter sequence is an increasing sequence such that the difference between every adjacent elements is one.

Predicates which appear in AF2 or AF3 type atoms are defined in terms of conditional rewriting rules and conditional inequalities.

(AF4) A linear inequality which represents the relation on the values of registers of protocol machines and the parameter values of messages in communication channels. For instance, " $V M_{A}=\operatorname{lastp}\left(\operatorname{subseq}\left(u_{A B},\{\mathrm{MIP}\}\right)\right)+1$ " is an AF4 type atom which states that the value of the register $V M$ of $P M_{A}$ is equal to the parameter of the last MIP message in $u_{A B}$ plus 1 at the global state under consideration.

For the disjunct $P_{i}$, let $s f_{A}\left(P_{i}\right)$ and $s f_{B}\left(P_{i}\right)$ denote the states of finite control of $P M_{A}$ and $P M_{B}$ respectively, specified in the AF1 type atom in $P_{i}$.

\subsection{An Example}

For example, let us consider a protocol $\Pi_{E X}=\left(P M_{A}, P M_{B}\right)$ based on the data transfer phase of OSI session protocol, and a formula $F_{E X}$ (Table 1,2.) 
Table 1: Registers of the protocol machines

\begin{tabular}{|c|l|}
\hline register name & \multicolumn{1}{c|}{ meaning } \\
\hline$V M_{A}, V M_{B}$ & the next serial number to be used \\
\hline$V A_{A}, V A_{B}$ & $\begin{array}{l}\text { the lowest serial number to which a synchronization } \\
\text { point confirmation is expected }\end{array}$ \\
\hline
\end{tabular}

Table 2: The definition of the actions of the protocol machines

\begin{tabular}{|c|}
$t a 1=\left(\mathrm{STA}_{713_{A}},\langle\mathrm{MIP}, s n\rangle, \mathrm{STA} 713_{A}, C_{t a 1}, R_{t a 1}\right) \in T_{A}$ \\
$C_{t a 1}:\left\{s n=V M_{A}\right\}$ \\
$R_{t a 1}\left(V M_{A}, V A_{A}\right)=\left(V M_{A}+1, V A_{A}\right)$ \\
\hline$t a 2=\left(\mathrm{STA}_{1} 3_{A},\langle\mathrm{MIA}, s n\rangle, \mathrm{STA} 713_{A}, \phi, R_{t a 2}\right) \in T_{A}$ \\
$R_{t a 2}\left(V M_{A}, V A_{A}\right)=\left(V M_{A}, s n+1\right)$ \\
\hline$t b 1=\left(\mathrm{STA} 713_{B},\langle\mathrm{MIA}, s n\rangle, \mathrm{STA} 713_{B}, C_{t b 1}, R_{t b 1}\right) \in T_{B}$ \\
$C_{t b 1}:\left\{V M_{B} \geq V A_{B}+1, V M_{B}=s n+1, s n \geq V A_{B}\right\}$ \\
$R_{t b 1}\left(V M_{B}, V A_{B}\right)=\left(V M_{B}, s n+1\right)$ \\
$t b 2=\left(\mathrm{STA}_{1} 3_{B},\langle\mathrm{MIP}, s n\rangle \mathrm{STA} 713_{B}, \phi, R_{t b 2}\right) \in T_{B}$ \\
$R_{t b 2}\left(V M_{B}, V A_{B}\right)=\left(V M_{B}+1, V A_{B}\right)$ \\
\hline
\end{tabular}

A protocol machine $P M_{A}$ can set up a synchronization point with a serial number $s n$ in data stream by sending a message $\langle$ MIP, $s n\rangle$ to the peer protocol machine $P M_{B}$. $P M_{A}$ increments a serial number of synchronization point by one on every transmission of message $\langle\mathrm{MIP}, s n\rangle$. The other protocol machine $P M_{B}$ can confirm to synchronization point by sending a message $\langle\mathrm{MIA}, s n\rangle . \quad P M_{B}$ does not need to respond every serial number. The serial number $s n$ of message $\langle$ MIA, $s n\rangle$ is equal to the maximum serial number among messages $\langle$ MIP,$x\rangle$ which $P M_{B}$ have already received.

Protocol machines $P M_{A}$ and $P M_{B}$ are defined as follows:

- The finite controls of $P M_{A}$ and $P M_{B}$ consist of one state STA $713_{A}$ and STA $713_{B}$ respectively. Both $P M_{A}$ and $P M_{B}$ have two integral registers. Table 1 tells intuitive description of those registers.

- $\Sigma_{A B}=\{\mathrm{MIP}\}, \Sigma_{B A}=\{$ MIA $\}$.

- Table 2 defines the set of actions of $P M_{A}$ and $P M_{B}$. For example, $t_{3}$ states the followings:

(i) If the two integral registers $V M_{B}$ and $V A_{B}$ satisfy the relation $V M_{B}>V A_{B}$, then $P M_{B}$ can send a message $\langle$ MIA, sn $\rangle$ such that $V M_{B}=s n+1, s n \geq V A_{B}$.

(ii) By executing the action the integral register $V A_{B}$ is incremented by one.

- The initial states of $P M_{A}$ and $P M_{B}$ are $\left\langle s f_{A}, 0,0\right\rangle$ and $\left\langle s f_{B}, 0,0\right\rangle$ respectively.

A human verifier is able to divide the reachability set of $\Pi_{E X}$ into 8 distinguished subsets by considering the truth values of the following statements: (1) There exists some messages whose message types are MIP, on the communication channel from $P M_{A}$ to $P M_{B} ;$ (2) There exist some messages whose message types are MIA, on the communication channel from $P M_{B}$ to $P M_{A}$; (3) $P M_{B}$ can send a message whose message type is MIA, that is equivalent to the inequality $V M_{B}>V A_{B}$. 
Table 3: Predicates and functions for a message sequence

\begin{tabular}{|l|l|}
\hline name & \multicolumn{1}{|c|}{ meaning } \\
\hline step $1(\alpha)$ & $\alpha$ is an increasing sequence by step 1 \\
st-inc $(\alpha)$ & $\alpha$ is a strictly increasing sequence \\
firstp $(\alpha)$ & the parameter value of the first message of $\alpha$ \\
lastp $(\alpha)$ & the parameter value of the last message of $\alpha$ \\
\hline
\end{tabular}

We define some predicates and functions to describe a formula. Table 3 shows an example of a definition of them. Table 4 shows an example of a logical formula that characterizes the above-mentioned eight subsets of the global states of $\Pi_{E X}$.

\subsection{A Verification Procedure}

In the verification method, for a given protocol $\Pi$ and a formula $F=P_{1} \vee P_{2} \vee \cdots \vee P_{n}$, $F$ is shown to be an invariant of $\Pi$ by the structural induction on event sequences of $\Pi$ as follows.

Inductive basis: Prove that every initial global state of $\Pi$ satisfies $F$.

Inductive step: Prove that

$$
\forall g s \in G S(F) \forall g s^{\prime}\left(g s \rightarrow g s^{\prime}\right)\left\{g s^{\prime} \in G S(F)\right\} .
$$

Observe that $G S(F)=G S\left(P_{1}\right) \cup G S\left(P_{2}\right) \cup \cdots \cup G S\left(P_{n}\right)$. Therefore, $\left({ }^{*} 1\right)$ is equivalent to

$$
\forall i(1 \leq i \leq n) \forall g s \in G S\left(P_{i}\right) \forall g s^{\prime}\left(g s \rightarrow g s^{\prime}\right) \exists j(1 \leq j \leq n)\left\{g s^{\prime} \in G S\left(P_{j}\right)\right\} .
$$

Thus $\left({ }^{*} 1\right)$ is proved by executing the following IS1 and IS2 for each $P_{i}(1 \leq i \leq n)$ and each action $t=\left(s f_{t}, d_{t}, s f_{t}^{\prime}, C_{t}, R_{t}\right) \in T_{X}$, where $X \in\{A, B\}$.

IS1 Identify every parameter $p$ and global state $g s \in G S\left(P_{i}\right)$ such that $(\langle d, p\rangle, X)$ is executable in $g s$.

This is examined by solving the simultaneous inequality consisting of $C_{t}$ and all AF4 type atoms in $P_{i}$.

IS2 For every executable parameter $n$ obtained by IS1, show

$$
\forall g s \in G S\left(P_{i}\right) \forall g s^{\prime}\left(g s \rightarrow \underset{X}{\left\langle d_{t}, n\right\rangle} g s^{\prime}\right) \exists j(1 \leq j \leq n)\left\{g s^{\prime} \in G S\left(P_{j}\right)\right\} .
$$

A verification system based on this method was developed (see [9].)

\section{A Method for Verifying Liveness}

\section{1 $Q$-liveness}

We formulate liveness property as $Q$-liveness defined as below.

Definition 4 For a protocol $\Pi$, let $G S_{Q}$ be the set of global states which satisfy a property $Q$. If $\forall g s \in R S_{\Pi} \exists g s^{\prime} \in G S_{Q}\left\{g s^{*} \rightarrow g s^{\prime}\right\}$ holds, the protocol $\Pi$ is said to be $Q$-live, where $R S_{\Pi}$ denotes the reachable set of $\Pi$. 
Table 4: A logical formula $E_{E X}$ for the protocol $\Pi_{E X}$ in 3.3

\begin{tabular}{|c|c|}
\hline $\begin{aligned} P_{1} & =\left\langle\operatorname{STA} 713_{A},{\text { STA } 713_{B}}\right\rangle \\
& \wedge\langle\varepsilon, \varepsilon\rangle \\
& \wedge V M_{A}=V M_{B} \\
& \wedge V A_{A}=V A_{B} \\
& \wedge V M_{A}=V A_{A} \\
& \wedge V M_{B}=V A_{B}\end{aligned}$ & $\begin{aligned} P_{2} & =\left\langle\text { STA } 713_{A},{\left.\text { STA } 713_{B}\right\rangle}\right\rangle \\
& \wedge\langle\varepsilon, \varepsilon\rangle \\
& \wedge V M_{A}=V M_{B} \\
& \wedge V A_{A}=V A_{B} \\
& \wedge V M_{A}>V A_{A} \\
& \wedge V M_{B}>V A_{B} ;\end{aligned}$ \\
\hline $\begin{aligned} P_{3} & =\left\langle\mathrm{STA} 713_{A}, \mathrm{STA}_{713_{B}}\right\rangle \\
& \wedge\left\langle\varepsilon, \mathrm{MIP} \cdot \mathrm{MIP}^{*}\right\rangle \\
& \wedge \text { step } 1\left(u_{A B}\right) \\
& \wedge V M_{A}=\text { lastp }\left(u_{A B}\right)+1 \\
& \wedge V M_{B}=\text { firstp }\left(u_{A B}\right) \\
& \wedge V A_{A}=V A_{B} \\
& \wedge V M_{A}>V A_{A} \\
& \wedge V M_{B}=V A_{B}\end{aligned}$ & $\begin{aligned} P_{4} & =\left\langle\mathrm{STA} 713_{A}, \mathrm{STA}_{713_{B}}\right\rangle \\
& \wedge\left\langle\varepsilon, \mathrm{MIP} \cdot \mathrm{MIP}^{*}\right\rangle \\
& \wedge \text { step } 1\left(u_{A B}\right) \\
& \wedge V M_{A}=\text { lastp }\left(u_{A B}\right)+1 \\
& \wedge V M_{B}=\text { firstp }\left(u_{A B}\right) \\
& \wedge V A_{A}=V A_{B} \\
& \wedge V M_{A}>V A_{A} \\
& \wedge V M_{B}>V A_{B}\end{aligned}$ \\
\hline $\begin{aligned} P_{5} & =\left\langle\mathrm{STA} \mathrm{ST}_{A}, \mathrm{STA}_{713_{B}}\right\rangle \\
& \wedge\left\langle\mathrm{MIA} \mathrm{MIA}^{*}, \varepsilon\right\rangle \\
& \wedge \text { st-inc }\left(u_{B A}\right) \\
& \wedge V M_{A}=V M_{B} \\
& \wedge V A_{B}=\operatorname{lastp}\left(u_{B A}\right)+1 \\
& \wedge V A_{A} \leq \operatorname{firstp}\left(u_{B A}\right) \\
& \wedge V M_{A}>V A_{A} \\
& \wedge V M_{B}=V A_{B}\end{aligned}$ & $\begin{aligned} P_{6} & =\left\langle\mathrm{STA} 713_{A}, \mathrm{STA}_{713_{B}}\right\rangle \\
& \wedge\left\langle\mathrm{MIA} \cdot \mathrm{MIA}^{*}, \varepsilon\right\rangle \\
& \wedge \text { st-inc }\left(u_{B A}\right) \\
& \wedge V M_{A}=V M_{B} \\
& \wedge V A_{B}=\operatorname{lastp}\left(u_{B A}\right)+1 \\
& \wedge V A_{A} \leq \operatorname{firstp}\left(u_{B A}\right) \\
& \wedge V M_{A}>V A_{A} \\
& \wedge V M_{B}>V A_{B}\end{aligned}$ \\
\hline $\begin{aligned} P_{7} & =\left\langle\mathrm{STA} \mathrm{ST}_{A}, \mathrm{STA} \mathrm{ST}_{B}\right\rangle \\
& \wedge\left\langle\mathrm{MIA} \mathrm{MIA}^{*}, \mathrm{MIP} \cdot \mathrm{MIP}^{*}\right\rangle \\
& \wedge \text { step } 1\left(u_{A B}\right) \\
& \wedge \text { st-inc }\left(u_{B A}\right) \\
& \wedge V M_{A}=\operatorname{lastp}\left(u_{A B}\right)+1 \\
& \wedge V M_{B}=\operatorname{firstp}\left(u_{A B}\right) \\
& \wedge V A_{B}=\operatorname{lastp}\left(u_{B A}\right)+1 \\
& \wedge V A_{A} \leq \operatorname{firstp}\left(u_{B A}\right) \\
& \wedge V M_{A}>V A_{A} \\
& \wedge V M_{B}=V A_{B}\end{aligned}$ & $\begin{aligned} P_{8} & =\left\langle\text { STA } 713_{A},{\left.\text { STA } 713_{B}\right\rangle}\right\rangle \\
& \wedge\left\langle\text { MIA } \text { MIA }^{*}, \text { MIP } \cdot \text { MIP }^{*}\right\rangle \\
& \wedge \text { step } 1\left(u_{A B}\right) \\
& \wedge \text { st-inc }\left(u_{B A}\right) \\
& \wedge V M_{A}=\text { lastp }\left(u_{A B}\right)+1 \\
& \wedge V M_{B}=\operatorname{firstp}\left(u_{A B}\right) \\
& \wedge V A_{B}=\text { lastp }\left(u_{B A}\right)+1 \\
& \wedge V A_{A} \leq \text { firstp }\left(u_{B A}\right) \\
& \wedge V M_{A}>V A_{A} \\
& \wedge V M_{B}>V A_{B}\end{aligned}$ \\
\hline
\end{tabular}

For example, if $Q$ states that some interested action $t$ is executable, then $Q$-liveness means that the action $t$ is eventually executed from any reachable state. If $Q$ characterizes normal completion, then $Q$-liveness means that the protocol can always normally complete.

For a protocol $\Pi$, a directed graph $R G_{\Pi}=\left(R S_{\Pi},\left\{\left(g s, g s^{\prime}\right) \mid g s \rightarrow g s^{\prime}\right\}\right)$ is called the reachability graph of $\Pi$, where $R S_{\Pi}$ denotes the reachability set of $\Pi$. If the reachability set $R S_{\Pi}$ is finite, $R G_{\Pi}$ is also finite. In such case, $Q$-liveness can be verified by exploring $R G_{\Pi}$ for a property $Q$ described as temporal logic formula. Such a verification method is 
called a model checking algorithm ${ }^{[4]}$. However, if the reachability set $R S_{\Pi}$ is infinite, it is impossible to apply the model checking algorithm for verifying $Q$-liveness of the protocol П.

In the rest of this paper, we consider a method for verifying $Q$-liveness of 2-ECFSMs in which $R S_{\Pi}$ is infinite. Let the reachability set $R S_{\Pi}$ be divided into finite numbers of distinguished subsets $G S_{1}, G S_{2}, \cdots, G S_{n}$. Let $V_{F}=\left\{v_{1}, v_{2}, \cdots, v_{n}\right\}$, where $n$ is the number of distinguished subsets introduced above. Let $G=\left(V_{F}, E\right)$ be a directed graph satisfying the following, where " $\Rightarrow$ " denotes an implication:

$$
\left(v_{i}, v_{j}\right) \in E \Rightarrow \forall g s \in G S_{i} \exists g s^{\prime} \in G S_{j}\left\{g s \rightarrow g s^{\prime}\right\} .
$$

Let $G S_{Q}=G S_{i}$ for some $i(1 \leq i \leq n)$, it is considerable to verify $Q$-liveness of $\Pi$ by exploring $G$. However, since the combination of " $\forall g s \in G S_{i}$ " and direct reachability in $\left({ }^{*} 2\right)$ is too strict, it seems that most executable transitions of given protocol may not be represented in such graph. Therefore, it is often the case that such a graph does not preserve the characteristics of given protocol.

Definition 5 For a protocol $\Pi$ and an invariant formula $F=P_{1} \vee P_{2} \vee \cdots \vee P_{n}$, a directed graph $G=\left(V_{F}=\left\{v_{i} \mid 1 \leq i \leq n\right\}, E\right)$ which satisfies the following is called a degenerated reachability graph of $\Pi$ :

$\left\{\right.$ there exists a path from $v_{i}$ to $v_{j}$ in $\left.G\right\} \Rightarrow \forall g s \in G S_{i} \exists g s^{\prime} \in G S_{j}\left\{g s^{*} \rightarrow g s^{\prime}\right\}$.

\subsection{A Degenerated Reachability Graph and $Q$-liveness}

Let $F=P_{1} \vee P_{2} \vee \cdots \vee P_{n}$ be an invariant of a protocol $\Pi$ and assume that $G S(F)$ contains no unsafe state. We will consider the verification of $Q$-liveness, where $Q$ is specified as $G S_{Q}=\bigcup_{i \in I_{Q}} G S\left(P_{i}\right)$, where $I_{Q}$ is a subset of the indexes $\{1 \leq i \leq n\}$ of the disjuncts $P_{i}$ of the invariant $F$. $\Pi$ holds:

Since $G S_{Q}=\bigcup_{i \in I_{Q}} G S\left(P_{i}\right)$, if the following formula holds, the $Q$-liveness of a protocol

$$
\forall i(1 \leq i \leq n) \forall g s \in G S\left(P_{i}\right) \exists j \in I_{Q} \exists g s^{\prime} \in G S\left(P_{j}\right)\left\{g s^{*} \rightarrow g s^{\prime}\right\} .
$$

In the following, if $g s^{\prime}$ is directly reachable from $g s$ by a sending event of $P M_{A}$ (or $P M_{B}$ ), we denote $g s \underset{\mathrm{A} \cdot \mathrm{s}}{\longrightarrow} g s^{\prime}$ (or $g s \underset{\mathrm{B} \cdot \mathrm{s}}{\longrightarrow} g s^{\prime}$ ). If $g s^{\prime}$ is directly reachable from $g s$ by a receiving event of $P M_{A}$ (or $P M_{B}$ ), we denote $g s \underset{\mathrm{A} \cdot \mathrm{r}}{\longrightarrow} g s^{\prime}$ (or $g s \underset{\mathrm{B} \cdot \mathrm{r}}{\longrightarrow} g s^{\prime}$ ). The transitive closure of the relations " $\overrightarrow{\mathrm{A} \cdot \mathrm{r}}$ " and " $\overrightarrow{\mathrm{B} \cdot \mathrm{r}}$ " are denoted by " $\overrightarrow{\mathrm{A} \cdot \mathrm{r}}$ " and $\stackrel{+}{\mathrm{B} \cdot \mathrm{r}}$ " respectively.

As a degenerated reachability graph of $\Pi$, we will consider the following directed graph $D R G=\left(V_{F}, E_{S} \cup E_{R}^{+}\right)$:

$$
\begin{aligned}
& V_{F}=\left\{v_{i} \mid 1 \leq i \leq n\right\} \\
& E_{S}=\left\{\left(v_{i}, v_{j}\right) \mid \forall g s \in G S\left(P_{i}\right) \exists g s^{\prime} \in G S\left(P_{j}\right)\left\{g s_{\overrightarrow{A \cdot \cdot}}^{\longrightarrow} g s^{\prime}\right\}\right\} \\
& \cup \quad\left\{\left(v_{i}, v_{j}\right) \mid \forall g s \in G S\left(P_{i}\right) \exists g s^{\prime} \in G S\left(P_{j}\right)\left\{g s_{\overrightarrow{\mathrm{B} \cdot \mathrm{s}}}^{\longrightarrow} g s^{\prime}\right\}\right\} ; \\
& E_{R}^{+}=\left\{\left(v_{i}, v_{j}\right) \mid \forall g s \in G S\left(P_{i}\right) \exists g s^{\prime} \in G S\left(P_{j}\right)\left\{g s \underset{\mathrm{A} \cdot \mathrm{s}}{\mathrm{A} \cdot \mathrm{r}} g s^{\prime}\right\}\right\} \\
& \cup\left\{\left(v_{i}, v_{j}\right) \mid \forall g s \in G S\left(P_{i}\right) \exists g s^{\prime} \in G S\left(P_{j}\right)\left\{g s \frac{7 \cdot \overrightarrow{\mathrm{B} \cdot \mathrm{r}}}{\longrightarrow} g s^{\prime}\right\}\right\} .
\end{aligned}
$$

If there exists a path from $v_{i}$ to $v_{j}$ in $D R G$, the following formula holds:

$$
\forall g s \in G S\left(P_{i}\right) \exists g s^{\prime} \in G S\left(P_{j}\right)\left\{g s^{*} \rightarrow g s^{\prime}\right\} .
$$


Since $F$ is an invariant in $\Pi$, if the following formula holds, then $\left({ }^{*} 3\right)$ holds:

$$
\forall i(1 \leq i \leq n) \exists j \in I_{Q}\left\{\text { there exists a path from } v_{i} \text { to } v_{j} \text { in } D R G\right\}
$$

\subsection{Constructing $D R G$}

In this subsection, we will describe a method for constructing a degenerated reachability graph $D R G=\left(V_{F}, E_{S} \cup E_{R}^{+}\right)$.

\subsubsection{Constructing $E_{S}$}

For $P_{i}, P_{j}(1 \leq i, j \leq n)$ and a sending action $t=\left(s f, d, s f^{\prime}, C, R\right)$ of $P M_{X}(X \in\{A, B\})$, if the following conditions (a) and (b) hold, then $\left(v_{i}, v_{j}\right) \in E_{S}$ :

(a) For every $g s=\left(s_{A}, s_{B}, c h_{B A}, c h_{A B}\right) \in G S\left(P_{i}\right)$, there exists a parameter value $p$ such that $\langle d, p\rangle$ is executable in $s_{X}$. This condition is written in the following(ES1)(ES3), where $C_{P_{i}}$ is a simultaneous inequality consisting of all AF4 type atoms in $P_{i}$. The set of inequalities in $C$ is divided into $C_{p}$ and $C_{\bar{p}}$, where $C_{p}$ consists of inequalities that contain the parameter variable $p C_{\bar{p}}$ consists of inequalities that does not contain $p$ :

(ES1) $s f_{X}\left(P_{i}\right)=s f$

(ES2) if the register values of $P M_{X}\left\langle p_{1}, p_{2}, \cdots, p_{r}\right\rangle$ satisfy $C_{P_{i}}$ then $\left\langle p_{1}, p_{2}, \cdots, p_{r}\right\rangle$ also satisfies $C_{\bar{p}}$;

(ES3) if the register values of $P M_{X}\left\langle p_{1}, p_{2}, \cdots, p_{r}\right\rangle$ satisfy $C_{P_{i}}$, then there exists a parameter value $p$ such that $\left\langle p_{1}, p_{2}, \cdots, p_{r}, p\right\rangle$ satisfy $C_{p}$ and $C_{P_{i}}$.

(b)

$$
\forall g s \in G S\left(P_{i}\right), p \forall g s^{\prime}\left(g s \rightarrow{ }_{X}^{\left\langle d_{t}, p\right\rangle} g s^{\prime}\right)\left\{g s^{\prime} \in G S\left(P_{j}\right)\right\} .
$$

$\left({ }^{*} 5\right)$ can be verified in a similar way to a part of the procedure described in [9]. (ES1) can be immediately verified from the values of $t$ and $P_{i}$. The procedure for verifying (ES2) and (ES3) will be described in 4.4. In the above way, several edges can be verified to be in $E_{S}$. Let $E_{S}^{\prime} \subseteq E_{S}$ be the set of such edges.

\subsubsection{Constructing $E_{R}^{+}$}

In the following discussion, for the AF2 type atom $\left\langle R_{B A}, R_{A B}\right\rangle$ in every $P_{i}(1 \leq i \leq n)$, we assume that $R_{B A}$ (or $R_{A B}$ ) specifies either a set consisting of only the empty sequence, that is $R_{B A}=\varepsilon$ (or $R_{A B}=\varepsilon$ ), or a set which does not contain the empty sequence.

We will consider the following graphs $G_{A, R}=\left(V_{F}, E_{A, R}\right)$ and $G_{B, R}=\left(V_{F}, E_{B, R}\right)$ whose edges express possibilities of transitions between two sets of global states by a reception by a specified protocol machine:

$E_{A, R}=\left\{\left(v_{i}, v_{j}\right) \mid \exists g s \in G S\left(P_{i}\right) \exists g s^{\prime} \in G S\left(P_{j}\right)\left\{g s \overrightarrow{\mathrm{A} \cdot \mathrm{r}} g s^{\prime}\right\}\right\}$
$E_{B, R}=\left\{\left(v_{i}, v_{j}\right) \mid \exists g s \in G S\left(P_{i}\right) \exists g s^{\prime} \in G S\left(P_{j}\right)\left\{g s \overrightarrow{\mathrm{B} \cdot \mathrm{r}} g s^{\prime}\right\}\right\}$.

For every $P_{i}, P_{j}(1 \leq i, j \leq n),\left(v_{i}, v_{j}\right) \in E_{A, R}$ (or $\left.\left(v_{i}, v_{j}\right) \in E_{B, R}\right)$ iff there exists a receiving action $t=\left(s f, d, s f^{\prime}, C, R\right)$ of $P M_{A}$ (or $P M_{B}$ ) satisfying the following (a) and (b): 
(a) There exists a global state $g s=\left(s_{A}, s_{B}, u_{B A}, u_{A B}\right) \in G S\left(P_{i}\right)$ and a parameter value $p$ such that $\langle d, p\rangle$ is executable, i.e. the following (ER1)-(ER3) holds, where $C_{P_{i}}$ is the simultaneous inequality consisting of all AF4 type atoms in $P_{i}$ and $\left\langle R_{B A, i}, R_{A B, i}\right\rangle$ is the AF2 type atom in $P_{i}$ :

(ER1) $s f_{A}\left(P_{i}\right)=s f\left(\right.$ or $\left.s f_{B}\left(P_{i}\right)=s f\right)$;

(ER2) $\exists u \in \Sigma_{B A}^{*}\left\{d \cdot u \in L\left(R_{B A, i}\right)\right\}$ (or $\exists u \in \Sigma_{A B}^{*}\left\{d \cdot u \in L\left(R_{A B, i}\right)\right\}$ );

(ER3) there exists register values $p_{1}, p_{2}, \cdots, p_{r}$ of $P M_{A}$ (or $P M_{B}$ ) and a parameter value $p$ of the receiving message $\langle d, p\rangle$ satisfying the simultaneous inequality consisting of $C_{P_{i}}$ and $C$.

(b)

$$
\exists g s \in G S\left(P_{i}\right), p, g s^{\prime} \in G S\left(P_{j}\right)\left\{g s \rightarrow \rightarrow_{A(o r B}^{\langle d, p\rangle} g s^{\prime}\right\} .
$$

(ER1) can be immediately determined from the values of $t$ and $P_{i}$. (ER2) and a necessary condition $\left({ }^{*} 6\right)$ ' of $\left({ }^{*} 6\right)$ also can be determined in a similar way to a part of the procedure described in [9]. The procedure for verifying (ER3) will be described in 4.4. We shall let $E_{A, R}^{\prime}$ (or $\left.E_{B, R}^{\prime}\right)$ be a set of edges $\left(v_{i}, v_{j}\right)$ such that (ER1), (ER2), (ER3), and (*6)' holds for some action $t$.

Let $\left\langle R_{B A, i}, R_{A B, i}\right\rangle$ be an AF2 type atom in $P_{i}$, consider the following the subsets of the node set $V_{F}$ of $D R G$ :

$V_{B A=\varepsilon}=\left\{v_{i} \mid R_{B A, i}=\varepsilon\right\}$

$V_{A B=\varepsilon}=\left\{v_{i} \mid R_{A B, i}=\varepsilon\right\}$.

Since we assumed that $G S(F)$ does not contain any unspecified reception state, for every global state $g s$, there exists a global state $g s^{\prime}$ reachable from $g s$ such that the communication channel from $P M_{B}$ to $P M_{A}$ is empty in $g s^{\prime}$, i.e. the following condition holds:

$$
\forall g s \in G S\left(P_{i}\right) \exists g s^{\prime} \in \bigcup_{v_{j} \in V_{B A=e}} G S\left(P_{j}\right)\left\{g s \underset{\mathrm{A} \cdot \mathrm{r}}{\stackrel{+}{\longrightarrow}} g s^{\prime}\right\} .
$$

Lemma 1 An edge $\left(v_{i}, v_{k}\right)$ is in $E_{R}^{+}$, if a node $v_{k}$ occurs on every path from $v_{i}$ to every $v_{j} \in V_{B A=\varepsilon}$ in $G_{A, R}^{\prime}=\left(V_{F}, E_{A, R}\right)$.

[Proof] Assume that a node $v_{k}$ occurs on every path from $v_{i}$ to every $v_{j} \in V_{B A=\varepsilon}$ in $G_{A, R}^{\prime}$. Then, the following formula holds:

$$
\begin{gathered}
\forall g s \in G S\left(P_{i}\right)\left\{\exists g s^{\prime} \in \bigcup_{v_{j} \in V_{B A=e}} G S\left(P_{j}\right)\left\{g s \frac{+}{\mathrm{A} \cdot \overrightarrow{\mathrm{r}}} g s^{\prime}\right\} \Rightarrow\right. \\
\left.\exists g s^{\prime} \in G S\left(P_{k}\right)\left\{g s \frac{+}{\mathrm{A} \cdot \mathrm{r}} g s^{\prime}\right\}\right\} .
\end{gathered}
$$

By $\left({ }^{*} 7\right)$ and (*8), $\forall g s \in G S\left(P_{i}\right) \exists g s^{\prime} \in G S\left(P_{k}\right)\left\{g s \underset{\mathrm{A} \cdot \mathbf{r}}{\stackrel{+}{\longrightarrow}} g s^{\prime}\right\}$ holds. Therefore, $\left(v_{i}, v_{k}\right) \in$ $E_{R}^{+}$.

We can obtain the same lemma for $E_{B, R}^{\prime}$.

By checking the sufficient condition in Lemma 1 for every pair of $v_{i}$ and $v_{j}$, several edges can be verified to be in $E_{R}^{+}$. Let $E_{R}^{\prime} \subseteq E_{R}^{+}$be a set of such edges. 


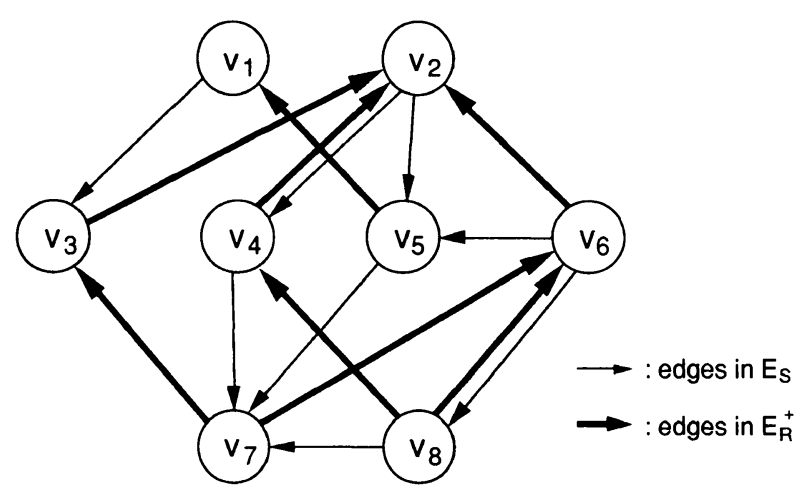

All self loops are omitted

Figure 1: $D R G_{\Pi}$ for $\Pi_{E X}$ and $F_{E X}$ in 3.3

\subsubsection{Exploring $D R G$}

Since $E_{S}^{\prime} \subseteq E_{S}, E_{R}^{\prime} \subseteq E_{R}^{+}$, if there exists a path from $v_{i}$ to $v_{j}$ in $\left(V_{F}, E_{S}^{\prime} \cup E_{R}^{\prime}\right)$, there also exists a path from $v_{i}$ to $v_{j}$ in $D R G=\left(V_{F}, E_{S} \cup E_{R}^{+}\right)$. Therefore, if the following holds, $\left({ }^{*} 4\right)$ and $Q$-liveness holds:

$$
\left.\forall i(1 \leq i \leq n) \exists j \in I_{Q} \text { \{there exists a path from } v_{i} \text { to } v_{j}\right\} .
$$

Figure 1 shows $\left(V_{F}, E_{S}^{\prime} \cup E_{R}^{\prime}\right)$ obtained by the above procedure on the protocol $\Pi_{E X}$ and invariant $F_{E X}$ in 3.3 . Since there exists a path from every node to $v_{i}(1 \leq i \leq n)$ in $\left(V_{F}, E_{S}^{\prime} \cup E_{R}^{\prime}\right), \Pi_{E X}$ is $P_{i}$-live for every $i(1 \leq i \leq n)$.

\subsection{Evaluating Presburger Formula}

Let $C$ be a simultaneous linear inequality with $m$ variables $x_{1}, x_{2}, \ldots x_{m}, c$ be a linear inequality whose variables are common with those of $C$, and $C_{y}$ be a simultaneous linear inequality whose variables are common with those of $C$ except only one variable $y$. The decision problems (ER3), (ES2) and (ES3) in 4.3 can be written in the following (S1), (S2), (S3) respectively, where $X$ denotes $\left\langle x_{1}, x_{2}, \ldots, x_{m}\right\rangle$ :

(S1) There exists an $S \in \mathcal{N}^{m}$ such that $X=S$ is a solution of $C$;

(S2) If $X=S\left(\in \mathcal{N}^{m}\right)$ is a solution of $C$, then $X=S$ satisfies $c$;

(S3) If $X=S\left(\in \mathcal{N}^{m}\right)$ is a solution of $C$, then there exists an $s \in \mathcal{N}$ such that $(X, y)=$ $(S, s)$ is a solution of a simultaneous inequality consisting of $C$ and $C_{y}$.

These problems can be expressed as formulae of Presburger arithmetic, which is known to be decidable ${ }^{[5]}$. However, $(\mathrm{S} 1)$ is equivalent to the general form of integer linear programing, which is known to be $\mathcal{N} \mathcal{P}$-complete ${ }^{[5]}$.

In the following, we consider a special case such that (S1),(S2) and (S3) can be efficiently solved. 
Definition 6 A linear inequality of the following form is called a difference constraint, where $x$ and $y$ are variables, and $q$ is a nonnegative integer:

$$
x-y \leq q .
$$

If every AF4 type atom in $F$ and every inequality in the action condition $C$ of every action of $P M_{A}$ or $P M_{B}$ are difference constraints, then every inequality in (EA3), (ES2), (ES3) is also a difference constraint. For the satisfiability problem of a simultaneous inequality consisting of difference constraints, which is called a system of difference constraints, the following method is known ${ }^{[11]}$.

\subsubsection{Solving a System of Difference Constraints}

For a system of difference constraints $C$ with $m$ variables, construct the weighted digraph $G_{C}=\left(V_{C}, E_{C}\right)$ as follows:

- $V_{C}=\left\{v_{1}, v_{2}, \cdots, v_{m}\right\}$

- $E_{C}=\left\{\left(v_{i}, v_{j}\right) \mid x_{j}-x_{i} \leq q\right.$ is a difference constraint in $\left.C\right\}$;

- For a difference constraint $x_{j}-x_{i} \leq q$ in $\mathrm{C}$, the weight $w\left(v_{i}, v_{j}\right)$ of the edges $\left(v_{i}, v_{j}\right)$ is $q$

Then the following lemmas hold ${ }^{[11]}$.

Lemma 2 A system of difference constraints has a solution $S \in \mathcal{N}^{m}$ iff $G_{C}$ contains no negative-weight cycles.

Lemma $3 x_{j}-x_{i}$ is bounded under the system of difference constraints $C$ iff $G_{C}$ contains a path from $v_{i}$ to $v_{j}$. If $G_{C}$ contains a path from $v_{i}$ to $v_{j}$, let $d\left(v_{i}, v_{j}\right)$ be the weight of the shortest path from $v_{i}$ to $v_{j}$, then the following are equivalent:

- There exists a solution of $C$ such that $x_{j}=s_{j}, x_{i}=s_{i}$;

- $s_{j}-s_{i} \leq d\left(v_{i}, v_{j}\right)$.

Bellman-Ford algorithm determines in $O\left(\left|V_{C}\right| \cdot\left|E_{C}\right|\right)$ whether a given $G_{C}$ contains no negative-weight cycles ${ }^{[11]}$. It also finds a shortest path and its weight of a given $G_{C}$ for any pair of source node and destination node.

\subsubsection{Evaluating a Class of Presburger Formula}

In the following of this section, we suppose that every inequality in (S1)-(S3) is a difference constraint and let $l$ and $l_{y}$ be the numbers of inequalities in $C$ and $C_{y}$ respectively.

Obviously, (S1) can be solved in $O(l m)$. Let the constraint $c$ in (S2) be " $x_{j}-x_{i} \leq q$ ". By determining whether there exists no solution for the system of difference constraints consisting of $C$ and the constraint " $x_{i}-x_{j} \leq-q-1$ ", which is the negation of $c$, (S2) can be solved in $O(l m)$.

Let $C_{-y}$ and $C_{+y}$ be the set of difference constraints in $C_{y}$ such that the coefficient of $y$ are " -1 " and " +1 " respectively. For $c^{\prime} \in C_{-y}$ and $c^{\prime \prime} \in C_{+y}$, let $E\left(C, c^{\prime}, c^{\prime \prime}\right)$ be the predicate which states the following:

$\forall S($ solution of $C) \exists s_{y}\left\{\left(S, s_{y}\right)\right.$ satisfies $c^{\prime}$ and $\left.c^{\prime \prime}\right\}$. 
Lemma 4 (S3) holds iff $E\left(C, c^{\prime}, c^{\prime \prime}\right)$ holds for every pair of $c^{\prime} \in C_{-y}$, and $c^{\prime \prime} \in C_{+y}$.

[Proof] The necessity is trivial. To show the sufficiency, suppose that (S3) does not hold, then there exists a solution $S$ of $C$ such that:

$$
\neg \exists s_{y}\left\{\left(S, s_{y}\right) \text { satisfies } C_{y}\right\} .
$$

Let $S=\left(s_{1}, s_{2}, \ldots, s_{m}\right)$ be such a solution. By assigning $S$ to $X$, every constraint $s_{j}-y \leq q$ in $C_{-y}$ gives a lower bound $s_{j}-q$ of $y$. Let $c^{\prime}$ be the strongest constraint among them. Similarly, by assigning $S$ to $X$, every constraint $y-s_{i} \leq q$ in $C_{+y}$ gives a upper bound $q+s_{i}$ of $y$. Let $c^{\prime \prime}$ be the strongest constraint among them. Then, $\left({ }^{*} 9\right)$ is equivalent to the followings:

$$
\neg \exists s_{y}\left\{\left(S, s_{y}\right) \text { satisfies } c^{\prime} \text { and } c^{\prime \prime}\right\} .
$$

Therefore, $E\left(C, c^{\prime}, c^{\prime \prime}\right)$ does not hold.

The above lemma states that (a) if there exists a solution of $C$, (S3) can be determined by determining $E\left(C, c^{\prime}, c^{\prime \prime}\right)$ for every pair of $c^{\prime} \in C_{-y}$ and $c^{\prime \prime} \in C_{+y}$, (b) otherwise (S3) vacuously holds.

Let $c^{\prime}$ be $x_{j}-y \leq q^{\prime}$ and $c^{\prime \prime}$ be $y-x_{i} \leq q^{\prime \prime}$.

Lemma $5 E\left(C, c^{\prime}, c^{\prime \prime}\right)$ holds iff there exists a path from $v_{i}$ to $v_{j}$ in $G_{C}$ and $d\left(v_{i}, v_{j}\right) \leq$ $q^{\prime}+q^{\prime \prime}$, where $d\left(v_{i}, v_{j}\right)$ is the weight of a shortest path from $v_{i}$ to $v_{j}$.

[Proof] Clearly, the following relation holds.

$$
x_{j}-x_{i} \leq q^{\prime}+q^{\prime \prime} \Leftrightarrow \exists y\left\{x_{j}-y \leq q^{\prime} \wedge y-x_{i} \leq q^{\prime \prime}\right\} .
$$

Suppose that $G_{C}$ does not contain a path from $v_{i}$ to $v_{j}$. It follows from Lemma 3 that there exists a solution $S$ of $C$ such that $s_{j}-s_{i}>q^{\prime}+q^{\prime \prime}$. By $\left({ }^{*} 11\right)$ we can see that $E\left(C, c^{\prime}, c^{\prime \prime}\right)$ is false.

Suppose that $d\left(v_{i}, v_{j}\right)>q^{\prime}+q^{\prime \prime}$. Consider $s_{j}$ and $s_{i}$ such that $d\left(v_{i}, v_{j}\right) \geq s_{j}-s_{i}>$ $q^{\prime}+q^{\prime \prime}$. By $\left({ }^{*} 11\right)$, there exists no $s_{y}$ such that $s_{j}-s_{y} \leq q^{\prime}$ and $s_{y}-s_{i} \leq q^{\prime \prime}$. On the other hand, it follows from Lemma 3 that there exists a solution of $C$ such that $x_{j}=s_{j}$ and $x_{i}=s_{i}$. Therefore, $E\left(C, c^{\prime}, c^{\prime \prime}\right)$ does not hold.

Suppose that $d\left(v_{i}, v_{j}\right) \leq q^{\prime}+q^{\prime \prime}$. It follows from Lemma 3 that $s_{j}-s_{i} \leq q^{\prime}+q^{\prime \prime}$ holds for each solution $\left\langle s_{1}, s_{2}, \ldots, s_{m}\right\rangle$ of $C$. By $\left({ }^{*} 11\right), E\left(C, c^{\prime}, c^{\prime \prime}\right)$ holds.

Since, the Bellman-Ford algorithm can determine the existence of a path between two nodes and calculate the weight of shortest paths between two nodes, $E\left(C, c^{\prime}, c^{\prime \prime}\right)$ can be determined in $O(l m)$. Consequently, the following theorem holds.

Theorem 1 (S3) can be determined in $O\left(\operatorname{lm}\left(l_{y}\right)^{2}\right)$.

\section{An Experimental Result}

To show the usefulness of the proposed method, we performed an experiment to verify liveness of a sample protocol using a verification system based on the above method. 


\subsection{A Verification System}

We developed a verification system by incorporating the procedures described in section 4 to the verification system described in [9].

An input to the verification system consists of the definition of protocol machines, properties of predicates on sequence of integers such as step 1 explained in Section 3.2 and Table 3, a logical formula $F=P_{1} \vee P_{2} \vee \ldots P_{n}$, and $I_{Q}$ which defines the property $Q$. A human verifier can use macro notations to describe logical formulae.

The verification system executes the procedures described in [9] and Section 4 to verify safety and liveness of a given protocol. The system also includes the efficient procedure described in 4.4 to determine a restricted form of Presburger formula.

The system is described in C, lex and yacc, and is executed on UNIX environment. The size of the source code of this system is about 16,300 lines.

\subsection{Extracting a Sample Protocol}

As a sample protocol of non-channel-bounded 2-ECFSMs, $\Pi_{\mathrm{Ses}}=\left(P M_{\mathrm{Ses}_{A}}, P M_{\mathrm{ses}_{B}}\right)$ was extracted from the data transfer phase of kernel, duplex, minor synchronize and major synchronize functional units from the OSI session protocol. For simplification, two tokens $m a$ and $m i$ are integrated into a token $m a-m i$.

For the protocol $\Pi_{\mathrm{ses}}$, two protocol machines $P M_{\mathrm{Ses}_{A}}$ and $P M_{\mathrm{Ses}_{B}}$ are isomorphic concerning to $S, \Sigma$, and $T$ but differ in those initial states, i.e. $P M_{\mathrm{Ses}_{A}}$ owns $m a-m i$ token while $P M_{\mathrm{Ses}_{B}}$ does not, at those initial states. The size of the states of finite controls and the numbers of registers of the protocol machines are 10 and 2 respectively. The number of message types used in the protocol is 12 . The number of actions of each protocol machine is 22 . In the definition of the actions of the machines, the action condition $C$ of every action can be given in the form of difference constraints.

\subsection{An Experimental Result}

At first, a human verifier devided a set of global states expected to be reachable into 144 subsets and described a logical formula $F_{S A F E}{ }^{1}$. The verification system showed that $F_{S A F E}$ is invariant of $\Pi_{\text {ses }}$ and $G S\left(F_{S A F E}\right)$ does not contain any unsafe state in $\Pi_{\text {ses. }}$. Hence, it can be concluded that $\Pi_{\text {ses }}$ is safe.

For verifying liveness, a human verifier divided $G S\left(P_{i}\right)$ for each disjunct $P_{i}$ of $F_{S A F E}$ into several distinguished subsets by the following criteria:

Is communication channel from $P M_{\mathrm{Ses}_{B}}$ to $P M_{\mathrm{Ses}_{A}}$ empty?; Is communication channel from $P M_{\mathrm{Ses}_{A}}$ to $P M_{\mathrm{Ses}_{B}}$ empty?;

Does a condition for executing of each action hold?

Finally, the human verifier devided the reachability set of $\Pi$ into 480 subsets, and described a formula $F_{L I V E}$ based on those subsets. The numbers of atomic formulae of each types AF1-AF4 in $F_{L I V E}$ are $30,30,46,471$ respectively. The macro processor unfolds $F_{L I V E}$ and obtains a disjunctive normal form $F_{L I V E}^{\prime}$ (equivalent to $F_{L I V E}$ ) consisting of 480 disjuncts. Both in $F_{S A F E}$ and $F_{L I V E}$, every AF4 type atom specified as difference

\footnotetext{
${ }^{1}$ For describing $F_{S A F E}$ or $F_{L I V E}$, a human verifier used 2 predicates and 2 defined functions, which are similar to those of [9].
} 
constraint. Since each action condition is also specified as difference constraints, every (ER3), (ES2) and (ES3) are determined by the procedure discussed in 4.4

At first, the verification system showed that $F_{L I V E}^{\prime}$ is an invariant of $\Pi_{\text {ses }}$ and $G S\left(F_{L I V E}^{\prime}\right)$ does not contain any unsafe state in $\Pi_{\text {ses. }}$. The verification system also showed $P_{i}$-liveness for every disjunct $P_{i}$ of $F_{L I V E}^{\prime}$. During the procedure of verifying liveness, the Bellman-Ford procedure were called 27,628 times. For the degenerated reachability graph $D R G=\left(V_{F_{L I V E}^{\prime}}, E_{S} \cup E_{R}^{+}\right)$constructed in the procedure of verifying liveness, $\left|V_{F_{L I V E}^{\prime}}\right|=$ $480,\left|E_{S}\right|=1,566$, and $\left|E_{R}^{+}\right|=1,300$.

The above experiments were executed on a UNIX workstation(NWS-5000, 64MB). The CPU time in the executions of verifying safety and liveness were 40 seconds and 60 seconds respectively.

\section{Conclusion}

In this paper, we formulated liveness property as $Q$-liveness and propose a method for verifying $Q$-liveness of communication protocol modeled as 2-ECFSMs. We also decribed a verification system based on the proposed method and a verification example extracted from OSI session protocol.

\section{References}

[1] Lin,F.J. et al.: "Protocol Verification Using Reachability Analysis: The State Space Explosion Problem and Relief Strategies", Proc. ACM SIGCOMM'87, pp.126135(1987).

[2] Brand,D. and Zafiropulo,P.: "On Communicating Finite-State Machines", JACM, vol.30, no.2, pp.323-342(1983).

[3] Yuang,M.C. and Kershebaum,A.: "Parallel Protocol Verification: The Two-Phase Algorithm" Proc. 9th PSTV, pp.339-353(1989-06).

[4] Clarke,E.M. et al.: "Automatic Verification of Finite-State Concurrent System Using Temporal Logic Specification", ACM Trans.PLS, vol.8, no.2, pp.244-263 (1986).

[5] Hopcroft,J.E. and Ullman,J.D. : "Introduction to Automata Theory, Languages, and Computation", Addison-Wesley (1979).

[6] Gouda, M.G.: "Closed Covers: to Verify Progress for Communicating Finite-State Machines", IEEE Trans. SE, vol.10, no.11, pp.846-855(1984-11).

[7] Pachl,J.: "Protocol Description and Analysis Based on a State Transition Model with Channel Expressions", Proc. 7th PSTV, pp.207-219(1987-05).

[8] Finkel,A.: "A New Class of Analyzable CFSMs with Unbounded FIFO Channels", Proc. 8th PSTV, pp.283-294 (1988).

[9] Higuchi,M. et al.: "A Verification Method via Invariant for Communication Protocols Modeled as Extended Communicating Finite-State Machines", IEICE Trans. Commun., vol.E-76B, no.11, pp.1363-1372 (1993-11).

[10] Higuchi,M. et al.: "A Verification Procedure via Invariant for Extended Communicating Finite-State Machines", Proc. of 4th Workshop on CAV pp.359-370(1992-07).

[11] Cormen,T.H., Leiserson,C.E. and Rivest,R.L.: "Introduction to Algorithms", The MIT Press, pp.539-543 (1990).

[12] ISO: "Basic Connection Oriented Session Protocol Specification", ISO 8327. 